\title{
Binding Site Models
}

for one, two, four, and sixteen binding modes. Individual grid points belong to: the Core defined by the overlap of all ligands (marked as c); the Interface representing the contour map (marked by the regression coefficients); and the Bulk (no mark).

\begin{tabular}{|c|c|c|c|c|c|c|c|c|c|}
\hline \multirow{2}{*}{$\begin{array}{c}\text { Grid } \\
\text { point } \\
\text { No. }\end{array}$} & \multirow[t]{2}{*}{ Coordinates $(\mathrm{x}, \mathrm{y}, \mathrm{z})$} & \multicolumn{2}{|c|}{$\begin{array}{l}1 \text { mode } \\
{\left[\times 10^{-2}\right]}\end{array}$} & \multicolumn{2}{|c|}{2 modes } & \multicolumn{2}{|c|}{4 modes } & \multicolumn{2}{|c|}{16 modes } \\
\hline & & ster & elect & ster & elect & ster & elect & ster & elect \\
\hline & & & & & & & & & \\
\hline 1 & $(-8.000,-6.000,-4.000)$ & -0.001 & 0.201 & & & & & & \\
\hline 2 & $(-6.000,-6.000,-4.000)$ & -0.001 & 0.278 & & & & & & \\
\hline 3 & $(-4.000,-6.000,-4.000)$ & -0.003 & 0.282 & & & & & & \\
\hline 4 & $(-2.000,-6.000,-4.000)$ & -0.004 & 0.193 & & & & & & \\
\hline 5 & $(0.000,-6.000,-4.000)$ & -0.002 & 0.093 & & & & & & \\
\hline 6 & $(2.000,-6.000,-4.000)$ & 0.000 & -0.171 & & & & & & \\
\hline 7 & $(4.000,-6.000,-4.000)$ & 0.001 & -0.626 & & & & & & \\
\hline 8 & $(6.000,-6.000,-4.000)$ & 0.000 & -0.801 & & & & & & \\
\hline 9 & $(8.000,-6.000,-4.000)$ & 0.000 & -0.657 & & & & & & \\
\hline 10 & $(-8.000,-4.000,-4.000)$ & -0.001 & 0.268 & & & & & & \\
\hline 11 & $(-6.000,-4.000,-4.000)$ & -0.004 & 0.442 & & & & & & \\
\hline 12 & $(-4.000,-4.000,-4.000)$ & -0.007 & 0.503 & & & & & & \\
\hline 13 & $(-2.000,-4.000,-4.000)$ & -0.009 & 0.373 & & & & & & \\
\hline 14 & $(0.000,-4.000,-4.000)$ & -0.005 & 0.430 & & & & & & \\
\hline 15 & $(2.000,-4.000,-4.000)$ & 0.002 & 0.190 & & & & & & \\
\hline 16 & $(4.000,-4.000,-4.000)$ & 0.004 & -0.843 & & & & & & \\
\hline 17 & $(6.000,-4.000,-4.000)$ & -0.001 & -1.261 & & & & & & \\
\hline 18 & $(8.000,-4.000,-4.000)$ & -0.001 & -0.959 & & & & & & \\
\hline 19 & $(-8.000,-2.000,-4.000)$ & -0.003 & 0.243 & & & & & & \\
\hline 20 & $(-6.000,-2.000,-4.000)$ & -0.006 & 0.436 & & & & & & \\
\hline 21 & $(-4.000,-2.000,-4.000)$ & -0.008 & 0.586 & & & & & & \\
\hline 22 & $(-2.000,-2.000,-4.000)$ & -0.008 & 0.584 & & & & & & \\
\hline 23 & $(0.000,-2.000,-4.000)$ & -0.005 & 1.009 & & & & & & \\
\hline 24 & $(2.000,-2.000,-4.000)$ & 0.001 & 1.291 & & & & & & \\
\hline 25 & $(4.000,-2.000,-4.000)$ & -0.003 & -0.019 & & & & & & \\
\hline 26 & $(6.000,-2.000,-4.000)$ & -0.008 & -1.211 & & & & & & \\
\hline 27 & $(8.000,-2.000,-4.000)$ & -0.003 & -1.133 & & & & & & \\
\hline 28 & $(-8.000,0.000,-4.000)$ & -0.005 & 0.127 & & & & & & \\
\hline 29 & $(-6.000,0.000,-4.000)$ & -0.012 & 0.170 & & & & & & \\
\hline 30 & $(-4.000,0.000,-4.000)$ & -0.011 & 0.353 & & & & & & \\
\hline 31 & $(-2.000,0.000,-4.000)$ & -0.006 & 0.678 & & & & & & \\
\hline 32 & $(0.000,0.000,-4.000)$ & -0.003 & 1.266 & & & & & & \\
\hline 33 & $(2.000,0.000,-4.000)$ & -0.003 & 1.894 & & & & & & \\
\hline 34 & $(4.000,0.000,-4.000)$ & -0.008 & 0.869 & & & & & & \\
\hline 35 & $(6.000,0.000,-4.000)$ & -0.009 & -1.015 & & & & & & \\
\hline
\end{tabular}




\begin{tabular}{|c|c|c|c|c|c|c|c|c|c|}
\hline \multirow{2}{*}{$\begin{array}{l}\text { Grid } \\
\text { point } \\
\text { No. }\end{array}$} & \multirow[t]{2}{*}{ Coordinates $(\mathrm{x}, \mathrm{y}, \mathrm{z})$} & \multicolumn{2}{|c|}{$\begin{array}{l}1 \text { mode } \\
{\left[\times 10^{-2}\right]}\end{array}$} & \multicolumn{2}{|c|}{2 modes } & \multicolumn{2}{|c|}{4 modes } & \multicolumn{2}{|c|}{16 modes } \\
\hline & & ster & elect & ster & elect & ster & elect & ster & elect \\
\hline & & & & & & & & & \\
\hline 36 & $(8.000,0.000,-4.000)$ & -0.003 & -1.211 & & & & & & \\
\hline 37 & $(-8.000,2.000,-4.000)$ & -0.006 & 0.074 & & & & & & \\
\hline 38 & $(-6.000,2.000,-4.000)$ & -0.015 & 0.091 & & & & & & \\
\hline 39 & $(-4.000,2.000,-4.000)$ & -0.016 & 0.274 & & & & & & \\
\hline 40 & $(-2.000,2.000,-4.000)$ & -0.006 & 0.634 & & & & & & \\
\hline 41 & $(0.000,2.000,-4.000)$ & -0.003 & 1.056 & & & & & & \\
\hline 42 & $(2.000,2.000,-4.000)$ & -0.005 & 1.339 & & & & & & \\
\hline 43 & $(4.000,2.000,-4.000)$ & -0.003 & 0.439 & & & & & & \\
\hline 44 & $(6.000,2.000,-4.000)$ & 0.002 & -1.007 & & & & & & \\
\hline 45 & $(8.000,2.000,-4.000)$ & 0.000 & -1.084 & & & & & & \\
\hline 46 & $(-8.000,4.000,-4.000)$ & -0.003 & 0.082 & & & & & & \\
\hline 47 & $(-6.000,4.000,-4.000)$ & -0.007 & 0.131 & & & & & & \\
\hline 48 & $(-4.000,4.000,-4.000)$ & -0.009 & 0.244 & & & & & & \\
\hline 49 & $(-2.000,4.000,-4.000)$ & -0.004 & 0.421 & & & & & & \\
\hline 50 & $(0.000,4.000,-4.000)$ & -0.003 & 0.688 & & & & & & \\
\hline 51 & $(2.000,4.000,-4.000)$ & -0.007 & 0.816 & & & & & & \\
\hline 52 & $(4.000,4.000,-4.000)$ & -0.006 & 0.309 & & & & & & \\
\hline 53 & $(6.000,4.000,-4.000)$ & 0.000 & -0.472 & & & & & & \\
\hline 54 & $(8.000,4.000,-4.000)$ & 0.001 & -0.644 & & & & & & \\
\hline 55 & $(-8.000,6.000,-4.000)$ & -0.001 & 0.082 & & & & & & \\
\hline 56 & $(-6.000,6.000,-4.000)$ & -0.002 & 0.120 & & & & & & \\
\hline 57 & $(-4.000,6.000,-4.000)$ & -0.003 & 0.177 & & & & & & \\
\hline 58 & $(-2.000,6.000,-4.000)$ & -0.002 & 0.264 & & & & & & \\
\hline 59 & $(0.000,6.000,-4.000)$ & -0.002 & 0.397 & & & & & & \\
\hline 60 & $(2.000,6.000,-4.000)$ & -0.003 & 0.447 & & & & & & \\
\hline 61 & $(4.000,6.000,-4.000)$ & -0.003 & 0.226 & & & & & & \\
\hline 62 & $(6.000,6.000,-4.000)$ & -0.001 & -0.126 & & & & & & \\
\hline 63 & $(8.000,6.000,-4.000)$ & 0.000 & -0.290 & & & & & & \\
\hline 64 & $(-8.000,-6.000,-2.000)$ & -0.001 & 0.298 & & & & & & \\
\hline 65 & $(-6.000,-6.000,-2.000)$ & -0.005 & 0.484 & & & & & & \\
\hline 66 & $(-4.000,-6.000,-2.000)$ & -0.011 & 0.486 & & & & & & \\
\hline 67 & $(-2.000,-6.000,-2.000)$ & -0.016 & 0.169 & & & & & & -0.206 \\
\hline 68 & $(0.000,-6.000,-2.000)$ & -0.010 & 0.049 & & & & & & \\
\hline 69 & $(2.000,-6.000,-2.000)$ & 0.003 & -0.407 & & & & & & \\
\hline 70 & $(4.000,-6.000,-2.000)$ & 0.009 & -1.589 & & & & & & \\
\hline 71 & $(6.000,-6.000,-2.000)$ & 0.003 & -1.698 & & & & & & \\
\hline 72 & $(8.000,-6.000,-2.000)$ & 0.000 & -1.088 & & & & & & \\
\hline 73 & $(-8.000,-4.000,-2.000)$ & -0.005 & 0.465 & & & & & & \\
\hline 74 & $(-6.000,-4.000,-2.000)$ & 0.019 & 1.182 & & & & & & \\
\hline 75 & $(-4.000,-4.000,-2.000)$ & 0.300 & 1.564 & & & & & -0.578 & \\
\hline
\end{tabular}




\begin{tabular}{|c|c|c|c|c|c|c|c|c|c|}
\hline \multirow{2}{*}{$\begin{array}{c}\text { Grid } \\
\text { point } \\
\text { No. }\end{array}$} & \multirow[t]{2}{*}{ Coordinates $(\mathrm{x}, \mathrm{y}, \mathrm{z})$} & \multicolumn{2}{|c|}{$\begin{array}{l}1 \text { mode } \\
{\left[\times 10^{-2}\right]}\end{array}$} & \multicolumn{2}{|c|}{2 modes } & \multicolumn{2}{|c|}{4 modes } & \multicolumn{2}{|c|}{16 modes } \\
\hline & & ster & elect & ster & elect & ster & elect & ster & elect \\
\hline 76 & $(-2.000,-4.000,-2.000)$ & 3.534 & 0.095 & -0.187 & & -0.138 & 0.144 & & \\
\hline 77 & $(0.000,-4.000,-2.000)$ & 0.117 & 0.519 & & & & & & \\
\hline 78 & $(2.000,-4.000,-2.000)$ & -0.086 & 0.740 & & & & & -0.039 & \\
\hline 79 & $(4.000,-4.000,-2.000)$ & -0.997 & -5.454 & 0.762 & & & & & \\
\hline 80 & $(6.000,-4.000,-2.000)$ & -0.003 & -4.430 & & & & & & \\
\hline 81 & $(8.000,-4.000,-2.000)$ & -0.004 & -1.905 & & & & & & \\
\hline 82 & $(-8.000,-2.000,-2.000)$ & -0.010 & 0.403 & & & & & & \\
\hline 83 & $(-6.000,-2.000,-2.000)$ & 0.292 & 1.433 & & & & & & \\
\hline 84 & $(-4.000,-2.000,-2.000)$ & 0.055 & 0.268 & & & & -0.391 & & \\
\hline 85 & $(-2.000,-2.000,-2.000)$ & \multicolumn{2}{|c|}{$\mathrm{c}$} & & 0.208 & & & & 1.429 \\
\hline 86 & $(0.000,-2.000,-2.000)$ & \begin{tabular}{|l|}
-0.089 \\
\end{tabular} & 2.368 & & & & & & \\
\hline 87 & $(2.000,-2.000,-2.000)$ & \multicolumn{2}{|c|}{$\mathrm{c}$} & & & & & & \\
\hline 88 & $(4.000,-2.000,-2.000)$ & -0.980 & 3.327 & & 0.406 & & & & \\
\hline 89 & $(6.000,-2.000,-2.000)$ & 1.225 & -5.279 & & & & & 0.059 & \\
\hline 90 & $(8.000,-2.000,-2.000)$ & -0.018 & -2.489 & & & & & & \\
\hline 91 & $(-8.000,0.000,-2.000)$ & -0.023 & 0.021 & & & & & & \\
\hline 92 & $(-6.000,0.000,-2.000)$ & 0.994 & -0.674 & & & & & 0.362 & \\
\hline 93 & $(-4.000,0.000,-2.000)$ & \multicolumn{2}{|c|}{$\mathrm{c}$} & \multicolumn{2}{|c|}{$\mathrm{c}$} & \multicolumn{2}{|c|}{$\mathrm{c}$} & & \\
\hline 94 & $(-2.000,0.000,-2.000)$ & 0.082 & 0.645 & & & & 0.213 & & \\
\hline 95 & $(0.000,0.000,-2.000)$ & \multicolumn{2}{|c|}{$\mathrm{c}$} & \multicolumn{2}{|c|}{$\mathrm{c}$} & \multicolumn{2}{|r|}{$\mathrm{c}$} & & \\
\hline 96 & $(2.000,0.000,-2.000)$ & 0.303 & 9.277 & & & & & & \\
\hline 97 & $(4.000,0.000,-2.000)$ & \multicolumn{2}{|c|}{$\mathrm{c}$} & \multicolumn{2}{|c|}{ c } & \multicolumn{2}{|l|}{$\mathrm{c}$} & & \\
\hline 98 & $(6.000,0.000,-2.000)$ & 6.124 & -5.608 & & -1.232 & 0.145 & & & \\
\hline 99 & $(8.000,0.000,-2.000)$ & -0.012 & -3.189 & & & & & & \\
\hline 100 & $(-8.000,2.000,-2.000)$ & -0.026 & -0.031 & & & & & & \\
\hline 101 & $(-6.000,2.000,-2.000)$ & 2.141 & -0.324 & & & & & -0.094 & \\
\hline 102 & $(-4.000,2.000,-2.000)$ & 2.193 & 0.112 & & -0.450 & & & & \\
\hline 103 & $(-2.000,2.000,-2.000)$ & \multicolumn{2}{|c|}{$\mathrm{c}$} & \multicolumn{2}{|r|}{$\mathrm{c}$} & & & & \\
\hline 104 & $(0.000,2.000,-2.000)$ & -0.010 & 0.213 & & 0.196 & & & & 0.213 \\
\hline 105 & $(2.000,2.000,-2.000)$ & \multicolumn{2}{|c|}{$\mathrm{c}$} & \multicolumn{2}{|c|}{$\mathrm{c}$} & & & & \\
\hline 106 & $(4.000,2.000,-2.000)$ & -1.977 & 0.940 & 0.139 & 0.825 & 0.060 & & & \\
\hline 107 & $(6.000,2.000,-2.000)$ & -3.034 & -7.229 & & & & & & \\
\hline 108 & $(8.000,2.000,-2.000)$ & 0.004 & -3.054 & & & & & & \\
\hline 109 & $(-8.000,4.000,-2.000)$ & -0.011 & 0.066 & & & & & & \\
\hline 110 & $(-6.000,4.000,-2.000)$ & -0.036 & 0.157 & & & & & & \\
\hline 111 & $(-4.000,4.000,-2.000)$ & 0.974 & 0.419 & & & & & & \\
\hline 112 & $(-2.000,4.000,-2.000)$ & -1.767 & 0.048 & 0.111 & & -0.136 & & & \\
\hline 113 & $(0.000,4.000,-2.000)$ & -0.016 & 1.231 & & & & & & \\
\hline 114 & $(2.000,4.000,-2.000)$ & 1.458 & 2.660 & & & & -0.334 & & \\
\hline 115 & $(4.000,4.000,-2.000)$ & 1.216 & 1.603 & & & & & & \\
\hline
\end{tabular}




\begin{tabular}{|c|c|c|c|c|c|c|c|c|c|}
\hline \multirow{2}{*}{$\begin{array}{c}\text { Grid } \\
\text { point } \\
\text { No. }\end{array}$} & \multirow[t]{2}{*}{ Coordinates $(\mathrm{x}, \mathrm{y}, \mathrm{z})$} & \multicolumn{2}{|c|}{$\begin{array}{l}1 \text { mode } \\
{\left[\times 10^{-2}\right]}\end{array}$} & \multicolumn{2}{|c|}{2 modes } & \multicolumn{2}{|c|}{4 modes } & \multicolumn{2}{|c|}{16 modes } \\
\hline & & ster & elect & ster & elect & ster & elect & ster & elect \\
\hline & & & & & & & & & \\
\hline 116 & $(6.000,4.000,-2.000)$ & 0.005 & -1.553 & & & & & & \\
\hline 117 & $(8.000,4.000,-2.000)$ & 0.005 & -1.399 & & & & & & \\
\hline 118 & $(-8.000,6.000,-2.000)$ & -0.003 & 0.086 & & & & & & \\
\hline 119 & $(-6.000,6.000,-2.000)$ & -0.007 & 0.139 & & & & & & \\
\hline 120 & $(-4.000,6.000,-2.000)$ & -0.011 & 0.176 & & & & & & \\
\hline 121 & $(-2.000,6.000,-2.000)$ & -0.005 & 0.220 & & & & & & \\
\hline 122 & $(0.000,6.000,-2.000)$ & -0.004 & 0.610 & & & & & & \\
\hline 123 & $(2.000,6.000,-2.000)$ & -0.017 & 1.012 & & & & & & \\
\hline 124 & $(4.000,6.000,-2.000)$ & -0.016 & 0.651 & & & & & & \\
\hline 125 & $(6.000,6.000,-2.000)$ & -0.003 & -0.206 & & & & & & \\
\hline 126 & $(8.000,6.000,-2.000)$ & 0.000 & -0.478 & & & & & & \\
\hline 127 & $(-8.000,-6.000,0.000)$ & -0.002 & 0.351 & & & & & & \\
\hline 128 & $(-6.000,-6.000,0.000)$ & -0.008 & 0.626 & & & & & & \\
\hline 129 & $(-4.000,-6.000,0.000)$ & -0.018 & 0.638 & & & & & 0.223 & \\
\hline 130 & $(-2.000,-6.000,0.000)$ & 0.029 & 0.067 & & & & & & \\
\hline 131 & $(0.000,-6.000,0.000)$ & -0.011 & -0.041 & & & & & -10.916 & \\
\hline 132 & $(2.000,-6.000,0.000)$ & 0.008 & -0.559 & & & & -0.564 & & 0.844 \\
\hline 133 & $(4.000,-6.000,0.000)$ & 0.015 & -2.432 & & & & & & \\
\hline 134 & $(6.000,-6.000,0.000)$ & 0.005 & -2.359 & & & & & & \\
\hline 135 & $(8.000,-6.000,0.000)$ & 0.000 & -1.328 & & & & & & \\
\hline 136 & $(-8.000,-4.000,0.000)$ & -0.008 & 0.604 & & & & & & \\
\hline 137 & $(-6.000,-4.000,0.000)$ & 1.637 & 2.220 & & & & & & \\
\hline 138 & $(-4.000,-4.000,0.000)$ & 0.564 & 0.355 & & -0.177 & & & & \\
\hline 139 & $(-2.000,-4.000,0.000)$ & \multicolumn{2}{|c|}{$\mathrm{c}$} & \multicolumn{2}{|c|}{$\mathrm{c}$} & \multicolumn{2}{|c|}{$\mathrm{c}$} & \multicolumn{2}{|c|}{$\mathrm{c}$} \\
\hline 140 & $(0.000,-4.000,0.000)$ & -0.184 & 11.452 & & & & -0.055 & & \\
\hline 141 & $(2.000,-4.000,0.000)$ & \multicolumn{2}{|c|}{$\mathrm{c}$} & \multicolumn{2}{|c|}{$\mathrm{c}$} & \multicolumn{2}{|r|}{$\mathrm{c}$} & & \\
\hline 142 & $(4.000,-4.000,0.000)$ & -1.331 & 11.975 & & 0.213 & & & & \\
\hline 143 & $(6.000,-4.000,0.000)$ & 0.034 & -9.177 & & & 0.577 & & & \\
\hline 144 & $(8.000,-4.000,0.000)$ & -0.007 & -2.568 & & & & & & \\
\hline 145 & $(-8.000,-2.000,0.000)$ & -0.016 & 0.524 & & & & & & \\
\hline 146 & $(-6.000,-2.000,0.000)$ & 0.374 & 1.486 & & & 0.198 & & & \\
\hline 147 & $(-4.000,-2.000,0.000)$ & \multicolumn{2}{|c|}{$\mathrm{c}$} & \multicolumn{2}{|c|}{$\mathrm{c}$} & \multicolumn{2}{|c|}{$\mathrm{c}$} & \multicolumn{2}{|c|}{$\mathrm{c}$} \\
\hline 148 & $(-2.000,-2.000,0.000)$ & \multicolumn{2}{|c|}{$\mathrm{c}$} & \multicolumn{2}{|c|}{$\mathrm{c}$} & \multicolumn{2}{|c|}{$\mathrm{c}$} & \multicolumn{2}{|c|}{$\mathrm{c}$} \\
\hline 149 & $(0.000,-2.000,0.000)$ & \multicolumn{2}{|c|}{$\mathrm{c}$} & \multicolumn{2}{|c|}{$\mathrm{c}$} & 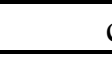 & c & c & 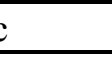 \\
\hline 150 & $(2.000,-2.000,0.000)$ & c & $\mathrm{c}$ & & $c$ & s & $\mathrm{c}$ & c & 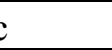 \\
\hline 151 & $(4.000,-2.000,0.000)$ & c & $\mathrm{c}$ & & $c$ & s & c & c & 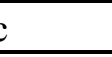 \\
\hline 152 & $(6.000,-2.000,0.000)$ & 2.183 & 1.515 & & 0.604 & & & & \\
\hline 153 & $(8.000,-2.000,0.000)$ & -0.030 & -3.554 & & & & & & \\
\hline 154 & $\left(\begin{array}{l}-8.000,0.000,0.000) \\
\end{array}\right.$ & 0.010 & -0.137 & & & & & & \\
\hline 155 & $\left(\begin{array}{lll}-6.000, & 0.000, & 0.000)\end{array}\right.$ & c & $\mathrm{c}$ & & c & - & c & & \\
\hline
\end{tabular}




\begin{tabular}{|c|c|c|c|c|c|c|c|c|c|}
\hline \multirow{2}{*}{$\begin{array}{c}\text { Grid } \\
\text { point } \\
\text { No. }\end{array}$} & \multirow[t]{2}{*}{ Coordinates $(\mathrm{x}, \mathrm{y}, \mathrm{z})$} & \multicolumn{2}{|c|}{$\begin{array}{l}1 \text { mode } \\
{\left[\times 10^{-2}\right]} \\
\end{array}$} & \multicolumn{2}{|c|}{2 modes } & \multicolumn{2}{|c|}{4 modes } & \multicolumn{2}{|c|}{16 modes } \\
\hline & & ster & elect & ster & elect & ster & elect & ster & elect \\
\hline 156 & $\left(\begin{array}{l}-4.000,0.000,0.000) \\
\end{array}\right.$ & \multicolumn{2}{|c|}{$\mathrm{c}$} & \multicolumn{2}{|c|}{$\mathrm{c}$} & \multicolumn{2}{|c|}{$\mathrm{c}$} & \multicolumn{2}{|c|}{$\mathrm{c}$} \\
\hline 157 & $\left(\begin{array}{lll}-2.000,0.000,0.000) \\
(\end{array}\right.$ & \multicolumn{2}{|c|}{$\mathrm{c}$} & \multicolumn{2}{|c|}{$\mathrm{c}$} & \multicolumn{2}{|c|}{$\mathrm{c}$} & \multicolumn{2}{|c|}{$\mathrm{c}$} \\
\hline 158 & $(0.000,0.000,0.000)$ & \multicolumn{2}{|c|}{$\mathrm{c}$} & \multicolumn{2}{|c|}{$\mathrm{c}$} & \multicolumn{2}{|c|}{$\mathrm{c}$} & \multicolumn{2}{|c|}{$\mathrm{c}$} \\
\hline 159 & $(2.000,0.000,0.000)$ & \multicolumn{2}{|c|}{$\mathrm{c}$} & \multicolumn{2}{|c|}{$\mathrm{c}$} & \multicolumn{2}{|c|}{$\mathrm{c}$} & \multicolumn{2}{|c|}{$\mathrm{c}$} \\
\hline 160 & $(4.000,0.000,0.000)$ & \multicolumn{2}{|c|}{$\mathrm{c}$} & s & $c$ & $\mathrm{c}$ & & & $c$ \\
\hline 161 & $(6.000,0.000,0.000)$ & & $\mathrm{c}$ & s & $c$ & $\mathrm{c}$ & & & \\
\hline 162 & $(8.000,0.000,0.000)$ & 0.095 & -5.302 & & & & & & \\
\hline 163 & $\left(\begin{array}{lll}-8.000, & 2.000, & 0.000)\end{array}\right.$ & 0.019 & -0.114 & & & & & & \\
\hline 164 & $\left(\begin{array}{lll}-6.000, & 2.000, & 0.000)\end{array}\right.$ & 1.787 & -0.001 & & & & & & \\
\hline 165 & $\left(\begin{array}{lll}-4.000, & 2.000, & 0.000)\end{array}\right.$ & & $\mathrm{c}$ & ( & $c$ & $\mathrm{c}$ & & & c \\
\hline 166 & $(-2.000,2.000,0.000)$ & & $\mathrm{c}$ & 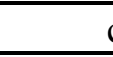 & $c$ & c & & & $\mathrm{c}$ \\
\hline 167 & $\left(\begin{array}{lll}0.000, & 2.000, & 0.000)\end{array}\right.$ & & $\mathrm{c}$ & 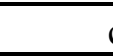 & $c$ & $\mathrm{c}$ & & & $\mathrm{c}$ \\
\hline 168 & $(2.000,2.000,0.000)$ & & $\mathrm{c}$ & ( & $c$ & c & & & c \\
\hline 169 & $(4.000,2.000,0.000)$ & $c$ & $\mathrm{c}$ & s & $c$ & $\mathrm{c}$ & & & $\mathrm{c}$ \\
\hline 170 & $(6.000,2.000,0.000)$ & 0.765 & 11.648 & & & & & & \\
\hline 171 & $(8.000,2.000,0.000)$ & -0.061 & -5.276 & & & & & & \\
\hline 172 & $(-8.000,4.000,0.000)$ & -0.019 & 0.060 & & & & & & \\
\hline 173 & $(-6.000,4.000,0.000)$ & 0.021 & 0.271 & & & -1.249 & & & \\
\hline 174 & $(-4.000,4.000,0.000)$ & -0.367 & -0.675 & & & 0.168 & & 0.215 & \\
\hline 175 & $(-2.000,4.000,0.000)$ & & $\mathrm{c}$ & - & $c$ & $\mathrm{c}$ & $c$ & & 0.201 \\
\hline 176 & $(0.000,4.000,0.000)$ & -0.342 & -0.144 & 0.073 & & 0.132 & & & \\
\hline 177 & $(2.000,4.000,0.000)$ & & $\mathrm{c}$ & s & $c$ & $\mathrm{c}$ & & & \\
\hline 178 & $(4.000,4.000,0.000)$ & 2.520 & 3.154 & 0.239 & & & & 0.091 & \\
\hline 179 & $(6.000,4.000,0.000)$ & -0.050 & -2.821 & & -1.248 & 0.708 & & & \\
\hline 180 & $(8.000,4.000,0.000)$ & 0.010 & -1.988 & & & & & & \\
\hline 181 & $\left(\begin{array}{lll}-8.000, & 6.000, & 0.000)\end{array}\right.$ & -0.004 & 0.089 & & & & & & \\
\hline 182 & $\left(\begin{array}{lll}-6.000,6.000, & 0.000) \\
\end{array}\right.$ & -0.013 & 0.155 & & & & & & \\
\hline 183 & $\left(\begin{array}{lll}-4.000, & 6.000, & 0.000)\end{array}\right.$ & -0.001 & 0.148 & & & & & & \\
\hline 184 & $\left(\begin{array}{lll}-2.000, & 6.000, & 0.000)\end{array}\right.$ & -0.062 & 0.013 & & & & & & \\
\hline 185 & $\left(\begin{array}{lll}0.000, & 6.000, & 0.000)\end{array}\right.$ & -0.007 & 0.717 & & & & & & \\
\hline 186 & $(2.000,6.000,0.000)$ & 0.044 & 1.626 & & & & 0.477 & & \\
\hline 187 & $(4.000,6.000,0.000)$ & 0.026 & 1.199 & & & & & & \\
\hline 188 & $(6.000,6.000,0.000)$ & -0.006 & -0.229 & & & & & & \\
\hline 189 & $(8.000,6.000,0.000)$ & 0.000 & -0.583 & & & & & & \\
\hline 190 & $(-8.000,-6.000,2.000)$ & -0.001 & 0.293 & & & & & & \\
\hline 191 & $(-6.000,-6.000,2.000)$ & -0.005 & 0.474 & & & & & & \\
\hline 192 & $(-4.000,-6.000,2.000)$ & -0.010 & 0.478 & & & & & & \\
\hline 193 & $(-2.000,-6.000,2.000)$ & -0.015 & 0.181 & & & & & & -0.206 \\
\hline 194 & $(0.000,-6.000,2.000)$ & -0.010 & 0.065 & & & & & & \\
\hline 195 & $(2.000,-6.000,2.000)$ & 0.003 & -0.379 & & & & & & \\
\hline
\end{tabular}




\begin{tabular}{|c|c|c|c|c|c|c|c|c|c|}
\hline \multirow{2}{*}{$\begin{array}{c}\text { Grid } \\
\text { point } \\
\text { No. }\end{array}$} & \multirow[t]{2}{*}{ Coordinates $(\mathrm{x}, \mathrm{y}, \mathrm{z})$} & \multicolumn{2}{|c|}{$\begin{array}{l}1 \text { mode } \\
{\left[\times 10^{-2}\right]}\end{array}$} & \multicolumn{2}{|c|}{2 modes } & \multicolumn{2}{|c|}{4 modes } & \multicolumn{2}{|c|}{16 modes } \\
\hline & & ster & elect & ster & elect & ster & elect & ster & elect \\
\hline 196 & & & & & & & & & \\
\hline 197 & $(6.000,-6.000,2.000)$ & 0.002 & -1.642 & & & & & & \\
\hline 198 & $(8.000,-6.000,2.000)$ & 0.000 & -1.066 & & & & & & \\
\hline 199 & $(-8.000,-4.000,2.000)$ & -0.004 & 0.454 & & & & & & \\
\hline 200 & $(-6.000,-4.000,2.000)$ & 0.010 & 1.128 & & & & & & \\
\hline 201 & $(-4.000,-4.000,2.000)$ & 0.190 & 1.476 & & & & & & \\
\hline 202 & $(-2.000,-4.000,2.000)$ & 1.869 & 0.052 & -0.187 & & -0.138 & & & \\
\hline 203 & $(0.000,-4.000,2.000)$ & 0.075 & 0.582 & & & & & & \\
\hline 204 & $(2.000,-4.000,2.000)$ & 0.027 & 0.751 & & & & & -0.039 & \\
\hline 205 & $(4.000,-4.000,2.000)$ & -0.672 & -4.905 & & & & & & \\
\hline 206 & $(6.000,-4.000,2.000)$ & -0.001 & -4.164 & & & & & & \\
\hline 207 & $(8.000,-4.000,2.000)$ & -0.004 & -1.855 & & & & & & \\
\hline 208 & $(-8.000,-2.000,2.000)$ & -0.010 & 0.387 & & & & & & \\
\hline 209 & $(-6.000,-2.000,2.000)$ & 0.185 & 1.316 & & & & & 0.085 & \\
\hline 210 & $(-4.000,-2.000,2.000)$ & 0.180 & 0.210 & & & & -0.391 & & \\
\hline 211 & $(-2.000,-2.000,2.000)$ & 0.078 & 6.263 & & 0.208 & & & & \\
\hline 212 & $(0.000,-2.000,2.000)$ & -0.053 & 2.348 & & & & & 0.162 & \\
\hline 213 & $(2.000,-2.000,2.000)$ & 0.102 & 7.240 & & 0.249 & & & & \\
\hline 214 & $(4.000,-2.000,2.000)$ & -0.722 & 1.674 & & & & & 0.142 & \\
\hline 215 & $(6.000,-2.000,2.000)$ & 0.862 & -4.867 & & & & & & \\
\hline 216 & $(8.000,-2.000,2.000)$ & -0.018 & -2.437 & & & & & & \\
\hline 217 & $(-8.000,0.000,2.000)$ & -0.023 & 0.014 & & & & & & \\
\hline 218 & $(-6.000,0.000,2.000)$ & 1.110 & -0.701 & 0.329 & & & & & \\
\hline 219 & $(-4.000,0.000,2.000)$ & $\mathrm{c}$ & $c$ & & $c$ & c & c & & \\
\hline 220 & $(-2.000,0.000,2.000)$ & 0.104 & 0.729 & & & & & & \\
\hline 221 & $(0.000,0.000,2.000)$ & $\mathrm{c}$ & $\mathrm{c}$ & & & $c$ & $c$ & & \\
\hline 222 & $(2.000,0.000,2.000)$ & 0.297 & 9.012 & & & & & & 0.188 \\
\hline 223 & $(4.000,0.000,2.000)$ & c & $\mathrm{c}$ & & $c$ & $\mathrm{c}$ & $c$ & & \\
\hline 224 & $(6.000,0.000,2.000)$ & 5.886 & -5.721 & 0.714 & -1.232 & 0.145 & & & \\
\hline 225 & $(8.000,0.000,2.000)$ & -0.012 & -3.191 & & & & & & \\
\hline 226 & $(-8.000,2.000,2.000)$ & -0.026 & -0.031 & & & & & & \\
\hline 227 & $(-6.000,2.000,2.000)$ & 2.312 & -0.305 & & & & & & \\
\hline 228 & $(-4.000,2.000,2.000)$ & 2.735 & 0.164 & & & & & & 0.238 \\
\hline 229 & $(-2.000,2.000,2.000)$ & ( & $\mathrm{c}$ & & $c$ & & & & \\
\hline 230 & $(0.000,2.000,2.000)$ & -0.005 & -0.086 & & 0.196 & & & & \\
\hline 231 & $(2.000,2.000,2.000)$ & c & c & & 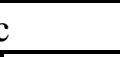 & & & & \\
\hline 232 & $(4.000,2.000,2.000)$ & -2.037 & 0.884 & 0.139 & 0.825 & 0.060 & & 0.138 & \\
\hline 233 & $(6.000,2.000,2.000)$ & -3.297 & -7.360 & & & & & & \\
\hline 234 & $(8.000,2.000,2.000)$ & 0.005 & -3.072 & & & & & & \\
\hline 235 & $(-8.000,4.000,2.000)$ & -0.011 & 0.067 & & & & & & \\
\hline
\end{tabular}




\begin{tabular}{|c|c|c|c|c|c|c|c|c|c|}
\hline \multirow{2}{*}{$\begin{array}{c}\text { Grid } \\
\text { point } \\
\text { No. }\end{array}$} & \multirow[t]{2}{*}{ Coordinates $(\mathrm{x}, \mathrm{y}, \mathrm{z})$} & \multicolumn{2}{|c|}{$\begin{array}{l}1 \text { mode } \\
{\left[\times 10^{-2}\right]}\end{array}$} & \multicolumn{2}{|c|}{2 modes } & \multicolumn{2}{|c|}{4 modes } & \multicolumn{2}{|c|}{16 modes } \\
\hline & & ster & elect & ster & elect & ster & elect & ster & elect \\
\hline & & & & & & & & & \\
\hline 236 & $(-6.000,4.000,2.000)$ & -0.037 & 0.164 & & & & & & \\
\hline 237 & $(-4.000,4.000,2.000)$ & 1.378 & 0.433 & & & & & & \\
\hline 238 & $(-2.000,4.000,2.000)$ & -2.712 & -0.067 & 0.111 & & & & & \\
\hline 239 & $(0.000,4.000,2.000)$ & -0.018 & 1.233 & & & & & & \\
\hline 240 & $(2.000,4.000,2.000)$ & 2.171 & 2.816 & & & & -0.334 & & \\
\hline 241 & $(4.000,4.000,2.000)$ & 1.752 & 1.780 & & & & & & \\
\hline 242 & $(6.000,4.000,2.000)$ & 0.005 & -1.548 & & & & & & \\
\hline 243 & $(8.000,4.000,2.000)$ & 0.005 & -1.403 & & & & & & \\
\hline 244 & $(-8.000,6.000,2.000)$ & -0.003 & 0.086 & & & & & & \\
\hline 245 & $(-6.000,6.000,2.000)$ & -0.008 & 0.139 & & & & & & \\
\hline 246 & $(-4.000,6.000,2.000)$ & -0.011 & 0.172 & & & & & & \\
\hline 247 & $(-2.000,6.000,2.000)$ & -0.005 & 0.207 & & & & & & \\
\hline 248 & $(0.000,6.000,2.000)$ & -0.005 & 0.612 & & & & & & \\
\hline 249 & $(2.000,6.000,2.000)$ & -0.017 & 1.043 & & & & & & \\
\hline 250 & $(4.000,6.000,2.000)$ & -0.017 & 0.682 & & & & & & \\
\hline 251 & $(6.000,6.000,2.000)$ & -0.004 & -0.197 & & & & & & \\
\hline 252 & $(8.000,6.000,2.000)$ & 0.000 & -0.477 & & & & & & \\
\hline 253 & $(-8.000,-6.000,4.000)$ & -0.001 & 0.197 & & & & & & \\
\hline 254 & $(-6.000,-6.000,4.000)$ & -0.001 & 0.271 & & & & & & \\
\hline 255 & $(-4.000,-6.000,4.000)$ & -0.002 & 0.278 & & & & & & \\
\hline 256 & $(-2.000,-6.000,4.000)$ & -0.003 & 0.198 & & & & & & \\
\hline 257 & $(0.000,-6.000,4.000)$ & -0.002 & 0.104 & & & & & & \\
\hline 258 & $(2.000,-6.000,4.000)$ & 0.000 & -0.148 & & & & & & \\
\hline 259 & $(4.000,-6.000,4.000)$ & 0.001 & -0.584 & & & & & & \\
\hline 260 & $(6.000,-6.000,4.000)$ & 0.000 & -0.763 & & & & & & \\
\hline 261 & $(8.000,-6.000,4.000)$ & 0.000 & -0.637 & & & & & & \\
\hline 262 & $(-8.000,-4.000,4.000)$ & -0.001 & 0.260 & & & & & & \\
\hline 263 & $(-6.000,-4.000,4.000)$ & -0.003 & 0.424 & & & & & & \\
\hline 264 & $(-4.000,-4.000,4.000)$ & -0.006 & 0.486 & & & & & & \\
\hline 265 & $(-2.000,-4.000,4.000)$ & -0.008 & 0.380 & & & & & & \\
\hline 266 & $(0.000,-4.000,4.000)$ & -0.005 & 0.441 & & & & & & \\
\hline 267 & $(2.000,-4.000,4.000)$ & 0.002 & 0.221 & & & & & & \\
\hline 268 & $(4.000,-4.000,4.000)$ & 0.003 & -0.744 & & & & & & \\
\hline 269 & $(6.000,-4.000,4.000)$ & -0.001 & -1.178 & & & & & & \\
\hline 270 & $(8.000,-4.000,4.000)$ & -0.001 & -0.925 & & & & & & \\
\hline 271 & $(-8.000,-2.000,4.000)$ & -0.003 & 0.233 & & & & & & \\
\hline 272 & $(-6.000,-2.000,4.000)$ & -0.006 & 0.411 & & & & & & \\
\hline 273 & $(-4.000,-2.000,4.000)$ & -0.007 & 0.558 & & & & & & \\
\hline 274 & $(-2.000,-2.000,4.000)$ & -0.007 & 0.585 & & & & & & \\
\hline 275 & $(0.000,-2.000,4.000)$ & -0.004 & 1.004 & & & & & & \\
\hline
\end{tabular}




\begin{tabular}{|c|c|c|c|c|c|c|c|c|c|}
\hline \multirow{2}{*}{$\begin{array}{c}\text { Grid } \\
\text { point } \\
\text { No. }\end{array}$} & \multirow[t]{2}{*}{ Coordinates $(\mathrm{x}, \mathrm{y}, \mathrm{z})$} & \multicolumn{2}{|c|}{$\begin{array}{l}1 \text { mode } \\
{\left[\times 10^{-2}\right]}\end{array}$} & \multicolumn{2}{|c|}{2 modes } & \multicolumn{2}{|c|}{4 modes } & \multicolumn{2}{|c|}{16 modes } \\
\hline & & ster & elect & ster & elect & ster & elect & ster & elect \\
\hline 276 & $(2.000,-2.000,4.000)$ & 0.000 & 1.287 & & & & & & \\
\hline 277 & $(4.000,-2.000,4.000)$ & -0.003 & 0.064 & & & & & & \\
\hline 278 & $(6.000,-2.000,4.000)$ & -0.008 & -1.130 & & & & & & \\
\hline 279 & $(8.000,-2.000,4.000)$ & -0.003 & -1.102 & & & & & & \\
\hline 280 & $(-8.000,0.000,4.000)$ & -0.005 & 0.121 & & & & & & \\
\hline 281 & $(-6.000,0.000,4.000)$ & -0.012 & 0.157 & & & & & & \\
\hline 282 & $(-4.000,0.000,4.000)$ & -0.011 & 0.340 & & & & & & \\
\hline 283 & $(-2.000,0.000,4.000)$ & -0.006 & 0.677 & & & & & & \\
\hline 284 & $(0.000,0.000,4.000)$ & -0.003 & 1.254 & & & & & & \\
\hline 285 & $(2.000,0.000,4.000)$ & -0.003 & 1.861 & & & & & & \\
\hline 286 & $(4.000,0.000,4.000)$ & -0.008 & 0.852 & & & & & & \\
\hline 287 & $(6.000,0.000,4.000)$ & -0.008 & -1.010 & & & & & & \\
\hline 288 & $(8.000,0.000,4.000)$ & -0.003 & -1.204 & & & & & & \\
\hline 289 & $(-8.000,2.000,4.000)$ & -0.006 & 0.072 & & & & & & \\
\hline 290 & $\left(\begin{array}{lll}-6.000, & 2.000, & 4.000)\end{array}\right.$ & -0.015 & 0.090 & & & & & & \\
\hline 291 & $(-4.000,2.000,4.000)$ & -0.016 & 0.275 & & & & & & \\
\hline 292 & $(-2.000,2.000,4.000)$ & -0.006 & 0.634 & & & & & & \\
\hline 293 & $(0.000,2.000,4.000)$ & -0.003 & 1.054 & & & & & & \\
\hline 294 & $(2.000,2.000,4.000)$ & -0.006 & 1.333 & & & & & & \\
\hline 295 & $(4.000,2.000,4.000)$ & -0.003 & 0.427 & & & & & & \\
\hline 296 & $(6.000,2.000,4.000)$ & 0.002 & -1.021 & & & & & & \\
\hline 297 & $(8.000,2.000,4.000)$ & 0.001 & -1.087 & & & & & & \\
\hline 298 & $(-8.000,4.000,4.000)$ & -0.003 & 0.082 & & & & & & \\
\hline 299 & $(-6.000,4.000,4.000)$ & -0.008 & 0.132 & & & & & & \\
\hline 300 & $(-4.000,4.000,4.000)$ & -0.010 & 0.243 & & & & & & \\
\hline 301 & $(-2.000,4.000,4.000)$ & -0.004 & 0.414 & & & & & & \\
\hline 302 & $(0.000,4.000,4.000)$ & -0.003 & 0.690 & & & & & & \\
\hline 303 & $(2.000,4.000,4.000)$ & -0.008 & 0.835 & & & & & & \\
\hline 304 & $(4.000,4.000,4.000)$ & -0.006 & 0.327 & & & & & & \\
\hline 305 & $(6.000,4.000,4.000)$ & 0.000 & -0.470 & & & & & & \\
\hline 306 & $(8.000,4.000,4.000)$ & 0.001 & -0.645 & & & & & & \\
\hline 307 & $(-8.000,6.000,4.000)$ & -0.001 & 0.082 & & & & & & \\
\hline 308 & 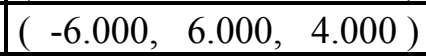 & -0.002 & 0.120 & & & & & & \\
\hline 309 & $(-4.000,6.000,4.000)$ & -0.003 & 0.175 & & & & & & \\
\hline 310 & $(-2.000,6.000,4.000)$ & -0.002 & 0.261 & & & & & & \\
\hline 311 & $(0.000,6.000,4.000)$ & -0.002 & 0.400 & & & & & & \\
\hline 312 & $(2.000,6.000,4.000)$ & -0.004 & 0.459 & & & & & & \\
\hline 313 & $(4.000,6.000,4.000)$ & -0.003 & 0.239 & & & & & & \\
\hline 314 & $(6.000,6.000,4.000)$ & -0.001 & -0.121 & & & & & & \\
\hline 315 & $(8.000,6.000,4.000)$ & 0.000 & -0.289 & & & & & & \\
\hline
\end{tabular}


- $9-$ 